\title{
Desenvolvimento de um jogo educativo para crianças com hemofilia
}

\author{
Roberta M. Matsunaga ${ }^{1,2}$, Regina L. de O. Moraes ${ }^{1}$, Marcos A. F. Borges ${ }^{1}$ \\ ${ }^{1}$ Faculdade de Tecnologia - Universidade Estadual de Campinas (FT/UNICAMP) - \\ Limeira - SP - Brasil. \\ ${ }^{2}$ Fundação Centro de Pesquisa e Desenvolvimento em Telecomunicações (CPqD) \\ robertamatsunaga@gmail.com, \{marcos.borges, regina\}@ft.unicamp.br
}

\begin{abstract}
This paper presents the Master's project of the student Roberta Mayumi Matsunaga. It is the development of an educational game for children with hemophilia. The goal is to encourage children with the disease to take appropriate attitudes towards the disease. Throughout the text is describing the process of interface development and the Project Management methodologies used.

Resumo. Este artigo apresenta o projeto de Mestrado da aluna Roberta Mayumi Matsunaga. Trata-se do desenvolvimento de um jogo educativo para crianças com hemofilia. O objetivo do jogo é estimular crianças portadoras da doença a tomar atitudes adequadas em relação à doença. Ao longo do texto será descrito o processo de desenvolvimento da interface e as metodologias de Gerenciamento de Projetos utilizadas.
\end{abstract}

\section{Introdução}

Jogos que têm a dupla finalidade - entreter e educar - são chamados Serious Games [Susi 2007]. Esse tipo de jogo se beneficia das estratégias conhecidas da indústria de jogos, que proporcionam entretenimento, para apoiar o aprendizado pelo jogador de algum tópico específico. Alguns dos objetivos de jogos educativos são: transmitir conhecimento, exercitar disciplinas e incitar comportamentos [Susi 2007].

O presente trabalho tem por objetivo apresentar o projeto de Mestrado em Tecnologia e Inovação da aluna Roberta Mayumi Matsunaga, realizado na Faculdade de Tecnologia da Universidade Estadual de Campinas (FT/UNICAMP). A aluna contou com a orientação do Professor Doutor Marcos Augusto Francisco Borges e coorientação da Professora Doutora Regina Lúcia de Oliveira Moraes. O projeto teve como intuito desenvolver o protótipo de um jogo educativo para crianças com hemofilia. A intenção é que o jogo seja um meio lúdico de informar, esclarecer e incentivar atitudes positivas das crianças com hemofilia em relação à doença com a qual irão conviver por toda a vida. A maior motivação do projeto é a carência de material educativo atrativo e de qualidade sobre a hemofilia que atinja a faixa etária pré-escolar e escolar.

O projeto é fruto de uma parceria da Faculdade de Tecnologia (FT) com a Unidade de Hemofilia do Hemocentro, ambos da Universidade Estadual de Campinas (UNICAMP). Os desenvolvedores contaram com o apoio da equipe multidisciplinar do 
Hemocentro, que é composta por médicos, fisioterapeutas, enfermeiros, pedagogos e psicólogos, todos especializados em hemofilia.

O desenvolvimento do protótipo, intitulado Hemotion, foi conduzido utilizando as boas práticas de gerenciamento de projetos, segundo o guia PMBOK. O time de desenvolvimento utilizou as técnicas da metodologia ágil Scrum na construção do protótipo. A estratégia pedagógica do jogo é inspirada na metodologia de ensino "Learning by Teaching". As fases de desenvolvimento do design, avaliação da usabilidade e validação do jogo contaram com a participação de crianças com hemofilia atendidas pelo Hemocentro.

O presente artigo irá trazer uma breve explicação sobre a doença foco do jogo, a hemofilia, e o objetivo do jogo educativo Hemotion (Seção 2); as metodologias de Gerenciamento de Projetos utilizadas ao longo do desenvolvimento são apresentadas na Seção 3; o processo de desenvolvimento do design do jogo é introduzido na seção 4, e, por fim, a conclusão é apresentada.

\section{A hemofilia e o jogo educativo Hemotion}

A hemofilia é uma coagulopatia (distúrbio relacionado à coagulação do sangue) hereditária recessiva que está diretamente relacionada ao sexo. Ela está presente em pessoas de todas as etnias. É consequência da falta ou defeito da produção dos fatores VIII ou IX de coagulação [Nunes et al. 2009]. Dados do Ministério da Saúde brasileiro (2005) mostram que a deficiência do fator VIII, que caracteriza a hemofilia A, corresponde a $85 \%$ dos casos; em contrapartida, a falta do fator IX, que caracteriza a hemofilia $\mathrm{B}$, corresponde a $15 \%$ dos casos. A quantidade destes fatores na circulação sanguínea é que determina a gravidade da doença, permitindo classificar a hemofilia em leve, moderada ou grave, de acordo com a quantidade de atividade do fator residual na circulação. O grau da hemofilia não sofre alterações durante a vida do indivíduo, não progride ou regride e não tem cura. Pacientes graves podem apresentar sangramentos recorrentes associados a pequenos traumas ou mesmo espontâneos [Vidal 2008]. A principal característica desta doença corresponde à dificuldade de coagulação frente a episódios hemorrágicos, que podem acometer tecidos profundos em qualquer local ou sistema, mas que tem maior prevalência em articulações e músculos. São as complicações musculoesqueléticas que correspondem a maior causa de deterioração na qualidade de vida de pessoas com hemofilia.

Como consequência da falta dos fatores de coagulação, a característica mais marcantes da hemofilia é a falta de controle dos sangramentos. Nesta situação, é necessária a administração endovenosa de concentrados contendo o fator deficiente. Estes sangramentos podem causar degeneração muscular, dor, osteoporose, deformidades, limitação funcional e, por consequência, invalidez [Hegeman et al. 2011]. As modalidades de tratamento clínico envolvem a reposição do fator deficiente, em quantidades necessárias para compensar a deficiência existente. Em alguns casos, o fator utilizado é confundido com um elemento estranho ao organismo, com os pacientes desenvolvendo anticorpos contra aquele fator, chamados inibidores. casos exigem uma abordagem diferente, dificultando consideravelmente o tratamento. 
Diante desta problemática, pessoas com hemofilia acabam por ter grandes restrições relativas a atividades físicas, a fim de evitar ferimentos e sangramentos. Em virtude do alto custo dos hemoderivados, em muitos países em desenvolvimento, o tratamento prevalente ainda se limita a infusão de fator nos episódios hemorrágicos, ou seja, sob demanda [Ozelo et al. 2012]. Já em países onde o tratamento profilático é prevalente, ou seja, há disponibilidade de fator para infusão periódica, as complicações musculoesqueléticas podem ser evitadas ou minimizadas significativamente. No Brasil o tratamento profilático se tornou realidade em dezembro de 2011, o Ministério da Saúde brasileiro implantou essa modalidade de tratamento para os pacientes que são portadores de hemofilia grave [Ministério da Saúde, 2011].

Diante do que foi exposto, pode-se concluir que crianças com hemofilia apresentam diversas restrições em suas atividades de vida diária, que interferem de forma significativa em sua qualidade de vida. Com o intuito de evitar traumas que possam levar a episódios hemorrágicos, as crianças com hemofilia, principalmente aquelas com hemofilia classificada como grave, são orientadas a evitar atividades físicas que envolvam risco de impacto e contato físico intenso, como no caso do futebol, das artes marciais e vários outros esportes. A abordagem deste assunto nem sempre é de fácil compreensão por parte da criança, sendo muitas vezes um desafio fazê-las compreender os riscos e suas complicações. Esclarecer pacientes e seus familiares sobre a hemofilia e o que decorre dela é um momento particularmente difícil, principalmente quando ocorre pela primeira vez na família. A utilização de meios de comunicação informativos pode se mostrar uma poderosa aliada nesta missão, principalmente quando envolvem figuras, vídeos e jogos que, segundo adolescentes com hemofilia, seriam mais envolventes do que páginas de textos [Sterling et al. 2012].

Todo o contexto exposto corrobora com a ideia de desenvolver um jogo educativo para crianças com hemofilia, que reforce conceitos e atitudes adequadas frente às diversas situações de vida enfrentadas desde a infância. A proposta do projeto de Mestrado foi desenvolver um jogo educativo onde crianças com hemofilia em idade escolar e pré-escolar aprendesse mais sobre a doença de forma interativa e motivadora. O jogo educativo, intitulado Hemotion, foi inspirado na metodologia de ensino "Learning by Teaching". Nessa metodologia, tutor e tutorado têm a mesma idade escolar e estão aprendendo o mesmo conteúdo. O tutor é então desafiado a ensinar o conteúdo ao tutorado. Dessa forma, o tutor irá aprender mais ensinando o próximo, e terá seus conhecimentos mais sedimentados. No Hemotion, de forma análoga, a criança com hemofilia representará o tutor do personagem do jogo, "ensinando" as condutas mais adequadas nas diferentes situações-problema que serão apresentadas.

A seção a seguir apresenta as metodologias de gerenciamento utilizadas para conduzir o projeto de mestrado e desenvolver o jogo educativo Hemotion.

\section{A utilização do Guia PMBOK e do framework Scrum}

O projeto de Mestrado foi conduzido utilizando as boas práticas de gerenciamento de projetos, segundo o guia PMBOK. Para a construção do protótipo do jogo, o time de desenvolvimento utilizou as técnicas da metodologia ágil Scrum. As próximas duas 
subseções trazem uma breve explicação da aplicação das duas metodologias no projeto aqui descrito.

\subsection{Utilização do Guia PMBOK}

O Guia PMBOK concentra as boas práticas identificadas em Gerenciamento de Projeto, que são amplamente reconhecidas. Utilizá-lo pode ser resumido na aplicação de conhecimento, habilidades, ferramentas e técnicas às atividades do projeto a fim de atender aos seus requisitos (PMI, 2008). Segundo o Guia PMBOK (2008) "Projeto é um empreendimento não repetitivo, caracterizado por uma sequência clara e lógica de eventos, com início, meio e fim, que se destina a atingir um objetivo claro e definido...". Tomando como base a descrição dada, o mestrado é considerado um projeto, pois, é algo temporário, ou seja, tem começo, meio e fim bem definidos e algo único, o que significa que seu resultado é singular. Como consequência, um projeto de mestrado pode ser gerenciado utilizando as técnicas de Gerenciamento de Projetos descrita no Guia PMBOK.

A condução do projeto de Mestrado foi realizada levando em consideração as premissas da quarta versão do Guia PMBOK. Todos os processos e boas práticas do Guia PMBOK que podiam ser adaptadas ao projeto de Mestrado foram implantados. Os membros do projeto adotaram também as nomenclaturas de importantes papéis desempenhados no projeto: o sponsor do projeto, pessoa responsável por gerenciar, é o professor orientador do mestrado; o cliente é o Hemocentro da Unicamp; o público alvo são as crianças com hemofilia que farão uso do jogo; os desenvolvedores do projeto são a aluna de Mestrado, a equipe multidisciplinar do Hemocentro e os bolsistas envolvidos no desenvolvimento do protótipo.

Os membros do projeto avaliaram a experiência da avaliação do Guia PMBOK para a condução de um projeto acadêmico como positiva e enriquecedora. Os grupos de processos do PMBOK permitiram logo no início do projeto uma visão mais abrangente do que precisaria ser realizado antes mesmo do projeto começar. Itens como escopo, qualidade, custo, entregas e atividades foram identificados logo no início do projeto, dando aos desenvolvedores maior visibilidade de como distribuir as tarefas ao longo do período do mestrado. Uma boa prática prevista no PMBOK é a construção de um Plano de Projeto, esse documento é o registro de como o projeto foi conduzido e todas as alterações sofridas. A construção e manutenção do Plano de Projeto permitiu uma melhor comunicação entre a aluna de Mestrado e o Professor orientador, uma vez que, toda alteração realizada pela aluna era enviada para revisão e aprovação do orientador.

Outra boa prática citada pelo PMBOK é a criação de pautas e atas para todas as reuniões de um projeto. Essa prática foi seguida a risca durante o projeto e auxiliou os membros do projeto na execução de todas as atividades que eram discutidas durante as reuniões, isso porque, tudo estava registrado e documentado. Utilizar pautas auxiliou os membros do projeto na condução de reuniões, pois todos os assuntos a serem abordados estavam pré - anotados. 


\subsection{Utilização do framework Scrum}

O framework Scrum é a implementação de metodologia ágil mais utilizada no desenvolvimento de sistemas [Varaschim, 2009]. O Scrum oferece um conjunto de boas práticas onde o gerenciamento do projeto é transparente para os usuários do modelo. Foi utilizado para gerenciar o desenvolvimento do jogo, buscando interações ágeis e um processo mais dinâmico. Assim como no Guia PMBOOK, o Scrum também determina a existência de alguns papéis para a condução do projeto. A nomenclatura utilizada e os papéis definidos foram: o Scrum Team era formado por quatro alunos de graduação e uma designer gráfico profissional; o papel de Product Owner foi desempenhado pelo professor orientador deste trabalho; o papel de Scrum Master foi desempenhado pela aluna de Mestrado.

A implantação do framework Scrum para a condução do desenvolvimento do jogo teve alguns pontos negativos e positivos. A maior problemática enfrentada pelos participantes do projeto foi a execução do Ritmo Sustentável. Nessa prática, a equipe mantém um ritmo de trabalho constante de 8 horas por dia, ou seja, 40 horas por semana. Essa prática é saudável para o gerenciamento do projeto, pois permite que o gerenciamento do tempo de execução das atividades seja mais preciso e confiável [Varaschim, 2009]. Diferente do ambiente de uma empresa, em um projeto acadêmico, os desenvolvedores não estão disponíveis para executar as tarefas em um período prédeterminado e constante. Horários de aula e férias semestrais foram um dos grandes ofensores do Ritmo Sustentável, pois, impossibilitou que o trabalho seguisse de forma constante.

Por outro lado, o framework Scrum auxiliou o Scrum Team a controlar o andamento das atividades. A ferramenta BurnDown Chart (gráfico que destaca as tarefas a realizar, em andamento e já realizadas), permitiu aos membros do time verificar de forma prática e rápida como estava o andamento do projeto e tomar ações preventivas para evitar atrasos. Outro ponto positivo para o projeto foi a divisão de todo o conjunto de tarefas em Sprints (ciclos de trabalhos que duram de duas a seis semanas), o que permitiu maior organização e visibilidade do andamento do projeto como um todo. Com o uso dessas duas ferramentas, o volume de tarefas ficou mais claro para todos os participantes.

A experiência mostrou que, faz-se necessário adaptar o framework Scrum para a sua utilização em projetos acadêmicos. Mesmo levando em conta os pontos negativos, o framework Scrum, mostrou ser uma das melhores opções para projetos acadêmicos. Isso porque, nas outras metodologias, como o Watterfall e RUP (Rational Unified Process), os períodos de interações são mais extensos. Um projeto que faz uso do RUP, por exemplo, tem ciclos de vida ou iterações que duram meses, ao contrário do Scrum onde interações duram semanas. Essa característica do Scrum permite entregas mais dinâmicas, pois, os ciclos têm duração curta.

\section{O processo de desenvolvimento da interface}

No começo do desenvolvimento do jogo os desenvolvedores identificaram a necessidade de envolver os usuários finais - crianças com hemofilia - na construção da interface. 
Foram então realizadas três dinâmicas durante o projeto de Mestrado que envolveu um conjunto de crianças com hemofilia. Como as dinâmicas envolveram a participação de seres humanos, foi necessário solicitar aprovação a um Comitê de Ética em Pesquisa, que garantiu e resguardou a integridade dos seres humanos envolvidos na pesquisa. A realização de todas as dinâmicas foi aprovada pelo Comitê de Ética em pesquisa da Faculdade de Ciências Médicas da Unicamp (FCM/UNICAMP) de acordo com a Resolução no 196/96 da CONEP (Comissão Nacional de Ética em Pesquisa).

As próximas subseções descrevem as três dinâmicas realizadas para apoiar o desenvolvimento da interface do Hemotion. A subseção 4.1 descreve a dinâmica Braindraw, a subseção 4.2 apresenta a dinâmica Avaliação Heurística Participativa e, por fim, a subseção 4.3 aborda a última dinâmica realizada, o Teste Beta.

\subsection{Primeira dinâmica: Braindraw}

O envolvimento de usuários finais no desenvolvimento do design de um sistema de computação é chamado Design Participativo [Walsh 2012]. Existem inúmeras técnicas que implementam Design Participativo: no presente projeto optou-se por utilizar a Braindraw. Braindraw é um tipo de dinâmica de prototipação participativa. Trata-se de um brainstorming gráfico feito nos moldes round-robin [Landauer e Prabhu 1998]. A abordagem desta dinâmica é interessante, pois o usuário pode desenhar o que está em sua mente sem receber críticas. Outra vantagem que se observou com a utilização dessa abordagem foi a aproximação dos desenvolvedores com as crianças com hemofilia, o que permitiu a identificação de alguns comportamentos dessas crianças em relação a doença.

$\mathrm{Na}$ dinâmica, cada participante realiza um design inicial na folha que recebeu. Decorrido um período de tempo pré-determinado, cada participante passa sua folha para o participante da esquerda e recebe o desenho do participante da sua direita. O participante deve então complementar o desenho que recebeu. No fim do período de tempo estabelecido, o participante repetirá a transição de folhas. Este procedimento é repetido até que todos os participantes tenham desenhado em todas as folhas, o que caracteriza o round-robin. Ao final, todos os participantes selecionam de forma democrática o desenho que mais lhes agradou. Com o desenho selecionado em mãos, os participantes realizam uma versão final.

Os desenvolvedores dividiram a dinâmica Braindraw em quatro rodadas. Em cada rodada, a criança tinham de fazer um desenho diferente. Os temas das rodadas eram: personagem principal do jogo que é portador de hemofilia; fator de coagulação; inibidor; cenário. Toda a interface do jogo foi desenvolvida levando-se em consideração os desenhos e ideias da dinâmica Braindraw [Matsunaga e Borges, 2012a].

- Alguns exemplos da colaboração das crianças para o desenvolvimento do design podem ser visualizados na Figuras 1 e 2. Na Figura 2 há um exemplo de colaboração das crianças no design do jogo. O personagem Fator do jogo foi baseado no desenho apresentado na parte central. 


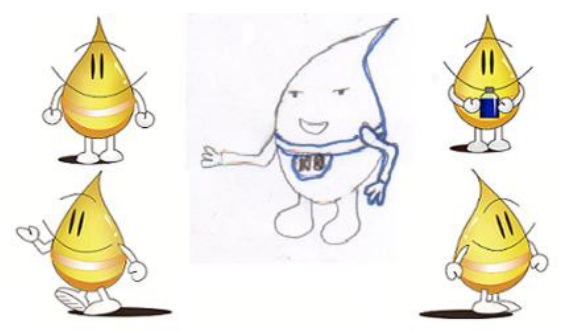

Figura 1. Compilação de alguns resultados da dinâmica para formação do Fator de Coagulação.

Na Figura 2, podemos observar outro exemplo de contribuição das crianças com hemofilia. Neste cenário, uma das crianças envolvida na dinâmica imaginou um dos cenários como sendo um vaso sanguíneo, onde o fator de coagulação entra em ação contra o inibidor.

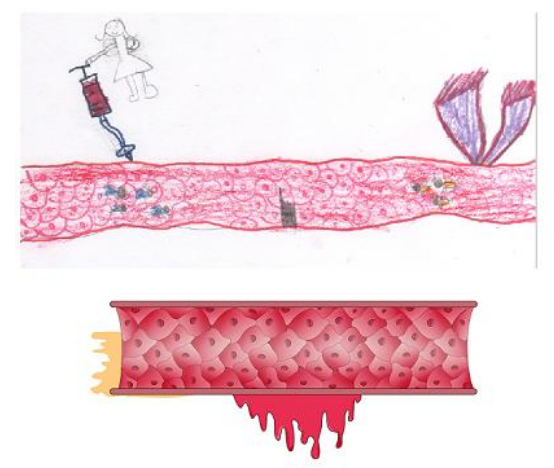

Figura 2. Compilação de alguns resultados da dinâmica para formação de um dos Cenários do jogo.

Como citado anteriormente, a dinâmica ajudou os desenvolvedores do jogo a situar alguns comportamentos típicos das crianças com hemofilia. Uma das observações mais importantes realizadas durante a dinâmica foi a opinião das crianças sobre o inibidor. Antes de realizar a dinâmica os desenvolvedores imaginavam que o inibidor era, para as crianças, algo negativo e prejudicial. Entretanto, durante as dinâmicas os desenvolvedores notaram que, para as crianças, o inibidor era algo 'bobo' que não sabia o que estava fazendo. Apesar de prejudicar potencialmente o tratamento, o inibidor não era visto como algo negativo, e sim, como um elemento que desconhece o quão prejudicial é para as pessoas com hemofilia.

A compilação dos resultados da dinâmica mostrou que alguns desenhos foram uma constante na contribuição das crianças. Como exemplos, pode-se enumerar: seringas, ambulâncias, medalhas, natação, amigo, etc. Dada essa observação, todos esses elementos foram incluídos como elementos do protótipo construído ao longo do projeto.

\subsection{Segunda dinâmica: Avaliação Heurística Participativa}

Assim como o desenvolvimento da interface foi realizada com o auxílio dos usuários finais do jogo, os desenvolvedores optaram por seguir a mesma abordagem com a avaliação da mesma. Foi realizada outra dinâmica com as crianças que permitiu aos desenvolvedores a análise da primeira interface do protótipo do jogo. 
A metodologia que foi empregada foi a Avaliação Heurística Participativa (AHP). A AHP tem por intuito mensurar a usabilidade de um sistema através do uso de regras específicas com a participação dos usuários finais. É uma técnica barata, rápida e efetiva de avaliar a interface de um sistema [Muller et al. 1998]. Vale ressaltar que usabilidade é o termo usado para definir a praticidade e conveniência com que as pessoas acessam um sistema, serviço ou produto [Chen e Macredie, 2005]. A avaliação é feita com base em princípios reconhecidos de interfaces de sistemas. Estes princípios são chamados de heurísticas.

Muller et al. (1998) definiu uma lista de heurísticas que são objetos de avaliação durante a Avaliação Heurística. Para a avaliação do jogo foram selecionadas algumas heurísticas que poderiam ser aplicáveis à avaliação de um jogo educativo. Os títulos do grupo de heurísticas selecionados são: 'Estado do sistema', 'Controle do usuário e liberdade', 'Consistência e relevância', 'Tarefas e apoio ao trabalho'. Mais detalhes sobre esses grupos de heurísticas podem ser consultados em [Muller, 1998, 16-18].

A dinâmica AHP contou com a participação de seis observadores adultos. Com base nas heurísticas estabelecidas, os observadores puderam avaliar como as crianças estavam se comportando. Um dos fatores observados foi a necessidade de o jogo ter mais interações com o usuário através de 'mini-games' (jogos interativos que representam brincadeiras, na primeira versão foi disponibilizado o 'mini-game' da bolinha de gude). Diante desta necessidade, os desenvolvedores passaram a incluir mais 'minigames', como o 'mini-game' do quebra-cabeça que pode ser visualizado na Figura 3.

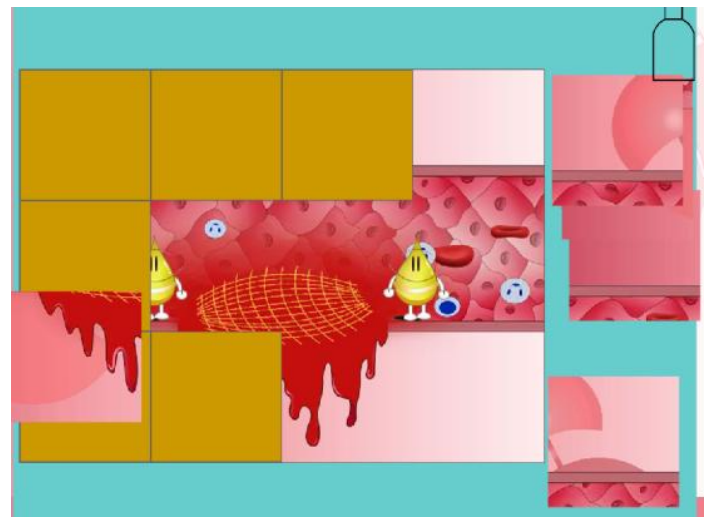

Figura 3. Mini-game do quebra-cabeça.

A dinâmica permitiu conhecer a opinião das crianças com hemofilia sobre o jogo. No mais, foi possível observar como foi a interação da criança com o jogo. Foram observados outros aspectos, tais como: influência do jogo nas crianças no contexto da hemofilia, mensagens passadas com o jogo, reações positivas ou negativas.

\subsection{Terceira dinâmica: Teste Beta}

A terceira e última dinâmica realizada foi o Teste Beta, que envolveu crianças com hemofilia na fase de teste do jogo. O teste beta é realizado nas instalações do usuário e tem por objetivo atestar a qualidade, confiabilidade e conformidade de um produto ou sistema. Teste Beta são também realizados em jogos do tipo MMORPG (Multi Massive Online Role-Playing Games) que comportam uma grande quantidade de jogadores interagindo e utilizando o jogo simultaneamente. Nesses casos, o teste beta é utilizado 
para verificar problemas de sobrecarga de servidores, problemas de autenticação e problemas de gerenciamento de contas. Novak (apud Neto, 2012) sustenta que "nesta fase todos os materiais já estão incorporados ao game e o processo de produção terminou. A fase beta tem como objetivo estabilizar o projeto e eliminar o maior número possível de defeitos antes que o produto comece a ser vendido".

Esta dinâmica teve um diferencial essencial para o projeto: contou com a participação de crianças que não eram portadoras de hemofilia. Essas crianças, no contexto da utilização do jogo, representavam o perfil de irmãos, amigos ou parentes de uma criança com hemofilia que utilizam o jogo para aprender mais sobre a doença. No total, participaram dessa dinâmica três crianças com hemofilia com idades entre 5 e 8 anos e quinze crianças que não eram portadoras de hemofilia com idades entre 5 e 6 anos. O resultado dessa dinâmica foi crucial para o projeto, pois foi possível verificar qual o entendimento que uma criança que não é portadora da hemofilia tem sobre a doença após jogar o Hemotion.

\section{Conclusão}

O presente desenvolvimento propõe formas de explorar os benefícios de aliar Tecnologia e Educação no apoio à Medicina. A obtenção de informações por meio de uma ferramenta tecnológica que envolve interação com o usuário torna o aprendizado um processo lúdico e motivador, o que pode ser muito positivo no caso específico da hemofilia, uma doença crônica rara que afeta indivíduos desde o nascimento. $\mathrm{O}$ aspecto lúdico é ainda mais importante quando o foco do projeto são crianças.

A construção do protótipo do Hemotion mostrou que a utilização de um jogo educativo para crianças com hemofilia pode ser uma ferramenta poderosa quando o objetivo é auxiliá-las a entender mais sobre a doença e suas consequências. Com o auxílio das duas últimas dinâmicas, há evidências que o protótipo pode ser útil no processo de construção de conhecimento sobre o assunto por crianças. Com base nestes resultados, os membros do time de desenvolvimento continuam no desenvolvimento do jogo, buscando construir uma nova versão do protótipo.

Durante o projeto de Mestrado foi desenvolvido também um Chatterbot especialista em hemofilia, o Dr. Remus Philius, que foi integrado ao Hemotion [Matsunaga et al., 2011; Matsunaga e Borges, 2012b]. Como próximos passos, estão previstos o uso do jogo no dia a dia do Hemocentro. Os participantes preveem também a internacionalização do jogo para outros idiomas como inglês e espanhol.

\section{Referências}

Chen, S. Y., \& Macredie R. D. (2005) "The assessment of usability of electronic shopping: A heuristic evaluation". International journal of Information Management, $25,516-32$.

Hegeman, A. K. et al.. (2001) "Perceived competence in children and adolescents with haemophilia: an explorative study". Haemophilia. v.17, p. 81-89. 
Landauer, T. K. and Prabhu, P. V. (1998) "Handbook of Human-Computer Interaction", New York, NY, USA: Elsevier Science Inc., 1998.

Matsunaga, R. M.; Matta, M. A. P.; Ozelo, M. C.; Borges, M. A. F. "Development of a Chatterbot specialist in Hemophilia Care". WFH 12th International Musculoskeletal Congress. Dubai. 2011.

Matsunaga, R. M.; Borges, M. A. F. "Developing a Serious Game for childreen with hemophilia". In: IADIS Multi Conference on Computer Science and Information Systems 2012, 2012, Lisboa. Proceedings of the IADIS International Conference Game and Entertainment Technologies. p. 77-80. 2012a.

Matsunaga, R. M.; Borges, M. A. F. "Developing a Chatterbot specialist in hemophilia". In: IADIS Multi Conference on Computer Science and Information Systems 2012, 2012, Lisboa. Proceedings of the IADIS International Conference Intelligent Systems and Agents. p. 169-172. 2012 b.

Ministério da Saúde (2005). "Manual de tratamento das coagulopatias hereditárias". Brasília. 39 p.

Ministério da Saúde (2011) "Política Nacional de Sangue e Hemoderivados". Brasília. 9p.

Muller, M. (2007), "Participatory design: The third space in HCI". In: J. Jacko and A. Sears (eds.), Handbook of HCI 2nd Edition.

Neto, D. P.; Santos, F. A. N. "Desenvolvimento de games: contribuição para a infografia interativa sob uma perspectiva e método de Design”. Human Factors in Design, v. 1, n 1.2012.

Nunes, A. A. et al.. (2009) "Qualidade de vida de pacientes hemofílicos acompanhados em ambulatório de hematologia". Revista Brasileira de Hematologia e Hemoterapia. v.31, n.6, p. 406-407.

Ozelo, M.C., Matta, M.A.P., Yang, R. (2012) "Meeting the challenges of haemophilia care and patient support in China and Brazil". Haemophilia, 18 (Suppl.5), 33-38.

Project Management Institute PMI (2008) Guia PMBOK. Quarta Edição.

Sterling L, Nyhof-young J, Blanchette VS and Breakey VR. (2012) "Exploring internet needs and use among adolescentes with haemophilia: a website development Project". Haemophilia, 18, 216-221.

Susi, T., Johanneson, M., Backlund, P. (2007) "Serious games - An overview". Technical report: HIS-IKI-TR-07-001, University of Skövde, Sweden.

Varaschim, J. D. "Implantando o SCRUM em um Ambiente de Desenvolvimento de Produtos para Internet”. Monografia em Ciência da Computação do Departamento de Informática da PONTIFÍCIA UNIVERSIDADE CATÓLICA DO RIO DE JANEIRO. 2009.

Vidal, T. O., Almeida, F. A. (2008). "Hemofilia na infầncia: o impacto da doença sobre a atuação dos pais na educação da criança.” , v.6, p.188-194.

Walsh, G. (2010) "Developing DisCo: A distributed co-design, on-line tool", HCIL2010-18 [Relatório Técnico]. College Park, MD: Human-Computer Interaction Lab. 